$$
\text { "tmcs-kolkova" — 2012/2/27 — 20:35 — page } 37 \text { — \#1 }
$$

\title{
A proposed application of Monte Carlo method in teaching probability
}

\author{
Mária Kolková, Jana Pócsová and Dušan Šveda
}

Abstract. Pupils' misconception of probability often results from lack of experience. Combining the concept of probability and statistics, the proposed application is intended for the teachers of mathematics at an elementary school. By reformulating the task in the form of an adventure, pupils examine a mathematical problem, which is too difficult for them to solve by combinatorial method. By recommending the simulation of the problem, we have sought to provide pupils with valuable experience of experimenting, recording and evaluating data.

Key words and phrases: simulation, experiment, estimation of probability.

ZDM Subject Classification: D83, F93, K53, K93.

\section{Introduction}

The common approach to teaching probability is based on the classical definition of probability and combinatorial calculations. For most pupils, however, such an approach is too difficult.

The Monte Carlo statistical method is based on the simulation of random variables, and the use of random numbers obtained by experimenting. According to Lane $([1])$, experimenting is something, which helps pupils understand the essence of probability. Therefore, we propose to apply the Monte Carlo method in teaching probability at an elementary school.

This work was partially supported by the grants VEGA 1/0168/10, 1/0390/10 and VVGS PF 50/2010/M.

Copyright (C) 2012 by University of Debrecen 
Our article focuses on the following task: "Throwing a dice ten times results in either a situation where each number has occurred at least once, or, a situation, where not all numbers have occurred. What is more likely?" Despite of our stress on the probability thinking, recognizing of the role of probability in nonmathematical context is another important subject of our article.

\section{Methodological part}

The formal denotation of the random trials $\left(\delta\right.$ and $\left.\delta_{s}\right)$ and the events related to them $\left(A, B, A_{s}\right.$ and $\left.B_{s}\right)$ in the proposed procedure are used so as to express them in accordance with their common mathematical usage. They do not need to be introduced to pupils.

The below problem represents a central part of our proposition:

A knight has to discover a message to save a kingdom. The message consists of six sentences. A long time ago, the message was known only to ten friends, all of whom wrote each sentence of the message on a separate scroll, and placed it in coffers. Each coffer contains the whole wording of the message. The ten coffers were hidden in an unknown castle.

The knight has found the castle, but he can only take one scroll from each coffer, read the sentence, and put the scroll back in the coffer. A guardian ensures that the knight takes only one scroll from each coffer.

If the knight manages to discover the whole message, he will save the kingdom. If not, he must think of a different way to discover the message. Will he manage to discover the whole message? Will he save the kingdom? Or, will he obtain an incomplete message? What is more likely?

We can answer the question more precisely only after we have identified the role of chance in this problem. Two random trials can be identified, one of them being a random selection of a sentence of the message from the coffer. This trial entails six equally probable outcomes. By repeating this trial ten times, a new random trial arises: random selection of sentences of the message from ten coffers. As a result we may arrive at $i$ different sentences of the message $(i \in 1,2, \ldots 6)$. The probabilities of these outcomes are not equal. The latter is the key random trial in this article and is denoted by $\delta$ (in accordance with [4]). 


$$
\text { "tmcs-kolkova" — 2012/2/27 — 20:35 — page } 39 \text { - \#3 }
$$

We have to focus on the probabilities of two mutually exclusive events given by the trial $\delta$ :

$A=\{\text { knight discovers the whole message }\}^{1}$

$B=\{k n i g h t$ fails to discover the whole message $\}$.

Pupils aged between 14 and 15 do not have the necessary mathematical skills and knowledge to solve this problem by calculation. Therefore, it is reasonable to introduce the simulation. We propose to use the Monte Carlo method. The Monte Carlo method will be explained with the help of six stages taken from [5] (p. 506-524).

To simulate the random trial $\delta$, an appropriate analogical trial needs to be produced, which can be performed by drawing lots, such as throwing a dice, or drawing a ball from a bag. The selection of a sentence from the coffer is random and does not depend on other choices, thus one of the appropriate analogical trials is a dice thrown 10-times. This trial is denoted $\delta_{s}$ (where $s$ stands for simulation). The path to discover the simulation comprises several steps, which can be expressed in the form of supporting questions:

Q1. How can we simulate a random selection of one sentence from the coffer (considering six outcomes with equal probability)?

Q2. How can we understand the outcome we obtain as part of the simulation by throwing the dice once?

Q3. How can we use the dice to simulate, which ten sentences will be discovered by the knight?

Q4. How can we infer whether the knight has discovered the whole message from the outcome of the ten throws of a dice?

The identification of an appropriate analogical trial is the subject matter of the first stage of the Monte Carlo method.

The second stage comprises the selection of a generator of random values (addressed in the first supporting question). This article considers a dice as the generator of random values.

The objective of the third stage of the Monte Carlo method is to determine the parameter we wish to find. The following events are included in the random trial $\delta_{s}$ :

$A_{s}=\{$ all six numbers occur in ten throws $\}$

$B_{s}=\{$ not all six numbers occur in ten throws $\}$,

${ }^{1}$ The events are stated in accordance with [4] in curly brackets.

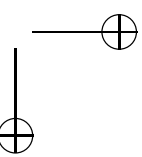




$$
\text { "tmcs-kolkova" — 2012/2/27 — 20:35 — page } 40 \text { - \#4 }
$$

which are analogical to the events $A$ and $B$ of the random trial $\delta$. We will examine the question which of the events $-A_{s}$ or $B_{s}$, is more likely.

The random trial $\delta$ needs to be simulated by repeating the random trial $\delta_{s}$ for as many times as necessary to arrive at the result reflecting the reality with the probability close to 1 . We propose that the trial $\delta_{s}$ is performed 250-times, which is a reasonable number of simulations to be performed during a lesson. ${ }^{2}$ As stated in the mathematical part below, repeating this trial 250-times represents a sufficient number of trials.

As part of the fourth stage, the outcomes of the simulation need to be recorded properly.

The fifth stage relates to the estimate of the sought parameter. It can be expected (see mathematical part below) that the event $B_{s}$ occurs more often than $A_{s}$ in the class. Based on the statistical data, we will conclude that the probability of the event $B_{s}$ is greater than the probability of $A_{s}$.

As part of the sixth and the last stage of the Monte Carlo method, the observations need to be interpreted. The probability of the event $A$ and $B$ is the same as the probability of the event $A_{s}$ and $B_{s}$. The probability of the event $A=$ $\{k n i g h t$ discovers the whole message $\}$ is smaller than the probability of the event $B=\{k n i g h t$ fails to discover the whole message $\}$.

\section{Mathematical part}

This part was included to make this article more comprehensive. It is not recommended to present it in the class.

First, we describe the calculation of the theoretical probability of the events $A_{s}$ and $B_{s}$. The outcome of a random $\operatorname{trial} \delta_{s}$ can be perceived as a mapping from the set $\{1,2, \ldots, 10\}$ to the set $\{1,2, \ldots, 6\}$. A mapping, which is not surjective, represents the throwing of a dice ten times, in that not all of the six numbers occur. Based on the principle of inclusion - exclusion, it can be determined that the number of all non-surjective mappings is 44030736 (for more details please refer inter alia to [3]). Hence, the probability of the event $B_{s}$ is $\frac{44030736}{6^{10}}$, i.e. approximately 0.728 , and the probability of the event $A_{s}$ is $1-\frac{44030736}{6^{10}}$, i.e. approximately 0.272 .

We propose that the trial $\delta_{s}$ is performed 250-times. Based on the Central Limit Theorem (cf. Riečan, Lámoš and Lenárt 1984, p. 111) it can be estimated

${ }^{2}$ We anticipate a class of 25 pupils with each student performing the trial $\delta_{s}$ 10-times.
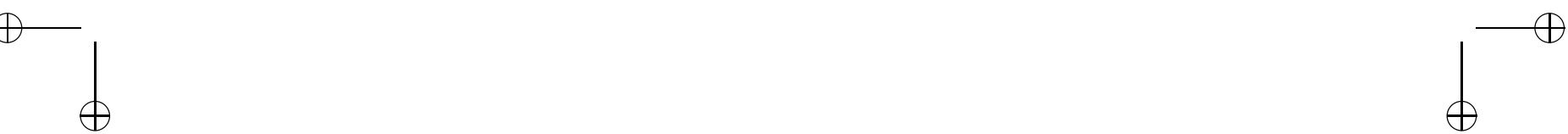


$$
\text { "tmcs-kolkova" — 2012/2/27 — 20:35 — page } 41 \text { — \#5 }
$$

that with a probability approximately 0.99 with this count of repetitions, the event $A_{s}$ occurs at least 50 -times, but no more than 86 -times. It is almost certain that at the end of the lesson it will be possible to come to the conclusion, that the probability of the event $A$ is smaller than the probability of the event $B$. Actually, we may conclude that the result 86 is statistically significant $(Z=-4.92, p<$ $0.01)$ to reject the hypothesis that the probability of the event $A_{s}$ and $B_{s}$ are identical, not to speak about the hypothesis that the probability of the event $A$ is greater (test for one proportion, cf. [2], p. 293 - 295).

\section{Conclusion}

The presented proposal allows pupils to reformulate the solved task to a mathematical problem, compare the probability of events using the simulation, and, finally, get familiarized with the statistical approach to probability.

The task to formulate the mathematical problem means that pupils had to:

- assign random trials analogical to the following trials: \{random selection of a sentence from the coffer $\}$ and \{random selection of sentences of a message from ten coffers\};

- assign analogical mathematical events to the events such as: \{knight discovers the whole message $\}$ and $\{$ knight fails to discover the whole message $\}$; and

- assign the mathematical questions analogical to the questions formulated when solving the problem: "Will the knight discover the whole message?", "Will the knight obtain an incomplete message?", "What is more likely?".

We have used the simulation to compare the probability of the events $\{k n i g h t$ discovers the whole message $\}$ and \{knight fails to discover the whole message $\}$. This article uses the Monte Carlo method.

The statistical approach was crucial in evaluating the statistical significance of the results obtained based on the statistical data. However, due to the limitations of pupils, the statistical approach can only be used on the level of intuition. The statistical approach was also present in performing the real random trial with a dice and this we consider one of the most important outcomes of our work. 


$$
\text { "tmcs-kolkova" — 2012/2/27 — 20:35 — page } 42 \text { — \#6 }
$$

42 M. Kolková, J. Pócsová and D. Šveda : A proposed application of Monte Carlo method...

\section{Acknowledgements}

The authors would like to thank Prof. Płocki for sharing with us the idea to use the Monte Carlo method in teaching probability. We would like to thank Daniel Klein, Ivan Žežula from Pavol Jozef Šafárik University in Košice in Slovakia and Jana Višňovská from The University of Queensland in Australia for their valuable comments which improved the final version of the paper. Great thanks belong also to schools which enable us to realize an experimental verification.

\section{References}

[1] A. Lane, Experimental probability in elementary school, Teaching Statistics 31, no. 2 (Summer 2009), $34-36$.

[2] D. S. Moore, The active practice of statistics, W. H. Freeman and Company, New York, 1997.

[3] L. Nguyen, The quantity of surjective mappings, [cited March 24, 2010], [online]. Available at http://unitaken.com/surjective/surjective.htm.

[4] A. Płocki, Pravdepodobnosť okolo nás: stochastika v úlohách a problémoch, Catholic University in Ružomberok, Ružomberok, 2007.

[5] A. Płocki, Stochastika dla nauczyciela : Rachunek prawdopodobienstwa, kombinatoryka $i$ statystyka matematyczna jako matematyka in statu nascendi, Wydawnictwo Naukowe NOVUM, Płock, 2007.

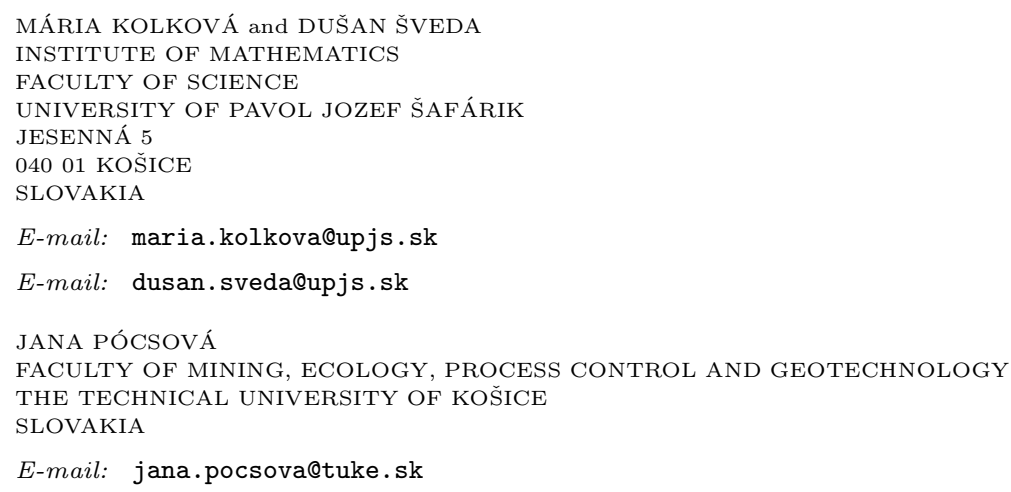

(Received September, 2010) 